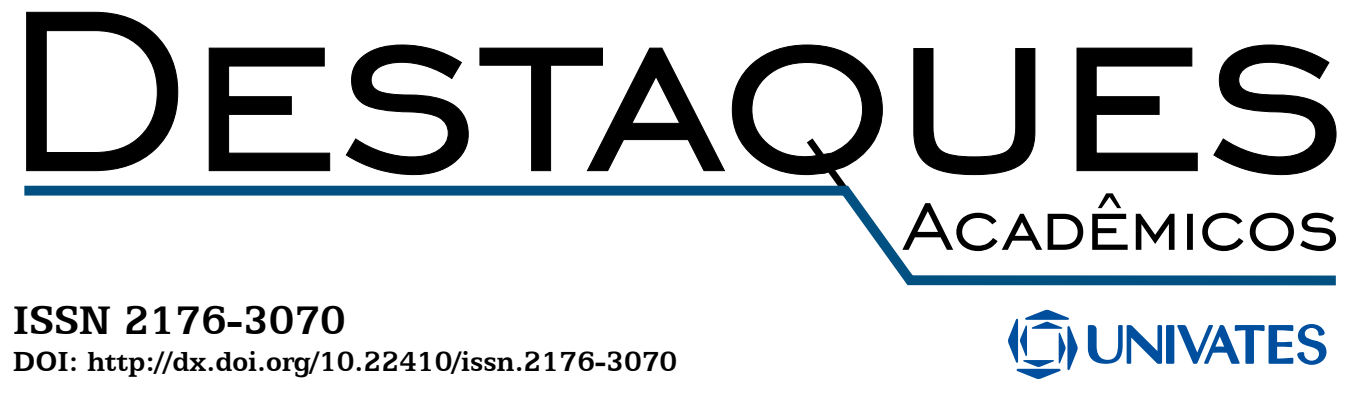

\title{
CIÊNCIAS BIOLÓGICAS E DA SAÚDE
}

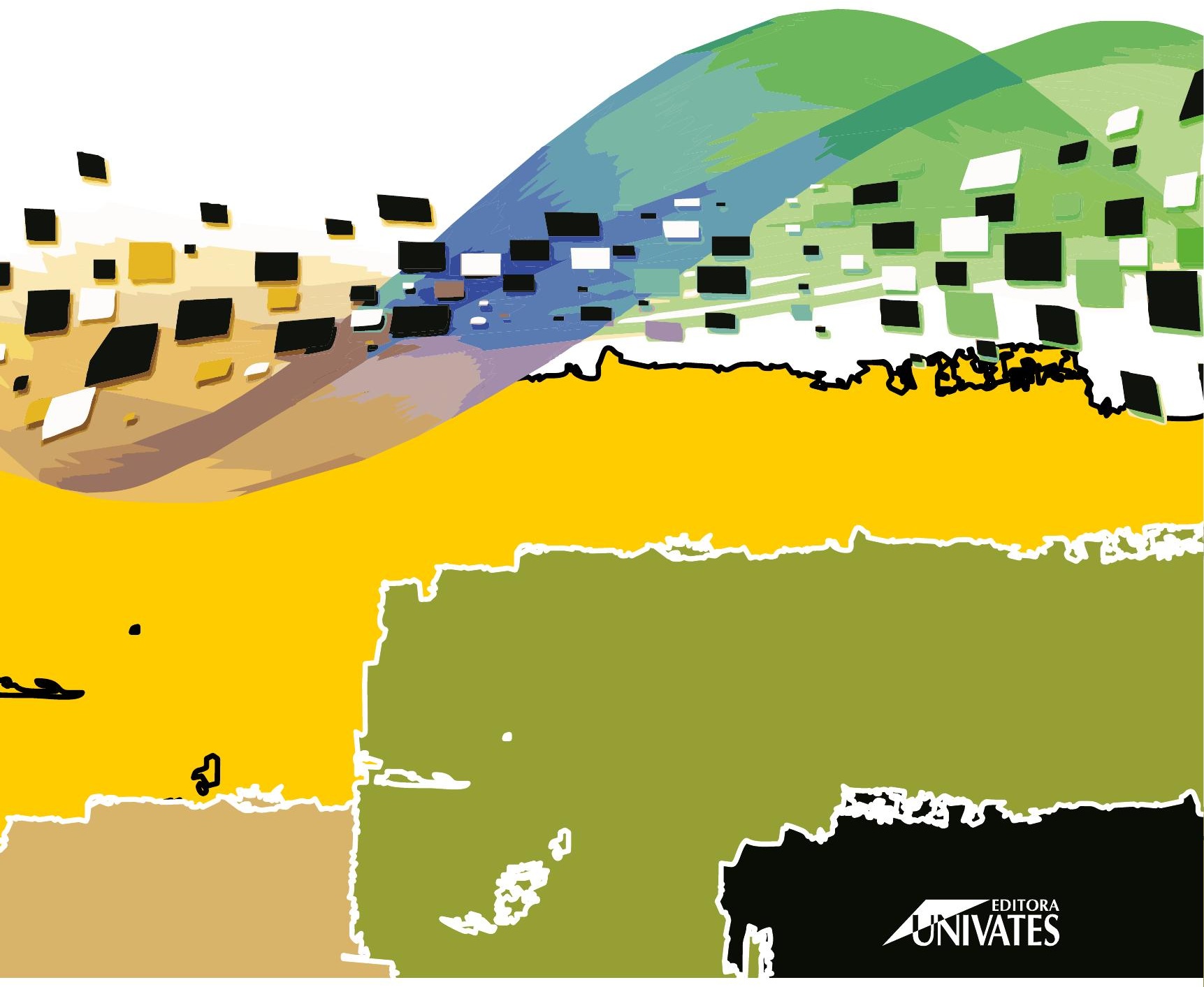




\section{(1) \\ UNIVATES}

Universidade do Vale do Taquari - Univates

Reitor: Prof. Me. Ney José Lazzari

Vice-Reitor e Presidente da Fuvates: Prof. Dr. Carlos Cândido da Silva Cyrne

Pró-Reitora de Pesquisa, Extensão e Pós-Graduação: Profa. Dra. Maria Madalena Dullius

Pró-Reitor de Ensino: Prof. Dr. Carlos Cândido da Silva Cyrne

Pró-Reitora de Desenvolvimento Institucional: Profa. Dra. Júlia Elisabete Barden

Pró-Reitor Administrativo: Prof. Me. Oto Roberto Moerschbaecher

\section{AINTIATIES}

Coordenação e Revisão Final: Ivete Maria Hammes

Editoração: Marlon Alceu Cristófoli

\section{Conselho Editorial da Editora Univates}

\section{Titulares}

Fernanda Rocha da Trindade

Marli Terezinha Quartieri

Rogério José Schuck

Fernanda Cristina Wiebusch Sindelar

\author{
Suplentes \\ Adriane Pozzobon \\ Ieda Maria Giongo \\ João Miguel Back \\ Alexandre André Feil
}

Avelino Talini, 171 - Bairro Universitário - Lajeado - RS, Brasil

Fone: (51) 3714-7024 / Fone/Fax: (51) 3714-7000

editora@univates.br / http://www.univates.br/editora

D476 Destaques Acadêmicos / Centro Universitário Univates - Vol. 1, no. 1 (2009) - Lajeado, RS: Ed. da Univates, 2017.

v. 9, n. 3, 2017.

Instituição mudou o nome para Universidade do Vale do Taquari - Univates em 2017.

Trimestral

ISSN 2176-3070

1. Ensino. 2. Ensino multidisciplinar. I. Centro Universitário Univates.

CDU: 378

Catalogação na publicação - Biblioteca da Univates

Os artigos são de exclusiva responsabilidade do(s) autor(es).

(C) Fundação Vale do Taquari de Educação e Desenvolvimento Social - FUVATES 


\section{DESTAOUES}

\section{Conselho Editorial da Revista}

Cristiane Antonia Hauschild

Giselda Veronice Hahn

João Miguel Back

Marlon Dalmoro

\section{Coordenadoras desta edição}

Andrea Horst

Lydia Christmann Espindola Koetz

\section{Pareceristas desta edição}

Alessandra Kerkhoff

Aline Gonçalves Cozer

Arlete Eli Kunz da Costa

Carlos Leandro Tiggemann

Cassia Regina Gotler Medeiros

Denise Fabiane Polonio

Eduardo Périco

Eduardo Sehnem

Fernanda Rocha da Trindade

Gabriela Kniphoff da Silva

Gabriela Kniphoff da Silva

Jairo Luis Hoerlle

Jonas Bernardes Bica

Kelly Mara Black

Liciane Diehl

Luísa Scheer Ely

Lydia Christmann Espindola Koetz

Marilucia V. dos Santos

Michele Beatriz Konzen

Mônica Jachetti Maciel

Olinda Maria de Fatima Lechmann Saldanha

Paula Michele Lohmann

Paulo Tadeu Campos Lopes

Temis Temis Jacques Bohrer

Vanderlei Biolchi 


\section{APRESENTAÇÃO}

Nesta edição, a Destaques Acadêmicos apresenta estudos e pesquisas realizados a partir do Centro de Ciências Biológicas e da Saúde, com temáticas que perpassam as questões ambientais e da saúde. Os artigos desta edição contemplam estudos provenientes de experiências de graduação, pós-graduação, extensão e pesquisas.

O produto final possui 24 artigos, que contemplam estudos da área de Psicologia, Nutrição, Enfermagem, Educação Física (bacharelado), Biomedicina, Farmácia e, inclusive estudo indígena, contribuindo para a ampliação do conhecimento acerca da região do Vale do Taquari e do Brasil.

O fomento a produção do conhecimento é uma das funções da universidade, seja por meio de atividades desenvolvidas no ensino, pesquisa, extensão ou pós-graduação, neste sentido, os estudos representam o conhecimento produzido por estudantes de graduação e pós-graduação e seus orientadores, promovendo o diálogo reflexivo, crítico e investigativo junto à comunidade externa e o mundo real.

Tomados pela visão da Universidade do Vale do Taquari - Univates, de "ser uma universidade de impacto social, cultural, econômico e tecnológico", esperamos que esta edição contribua para as discussões entre estudantes e profissionais das diversas áreas de formação, tendo a certeza de que a produção do conhecimento ocorre a partir da relação dialógica entre os pares.

Boa leitura.

Profa. Dra. Andréa Horst Profa. Dra. Lydia Christmann Espindola Koetz Coordenadoras desta edição 


\section{SUMÁRIO}

DENÚNCIA DE UM OLHAR FALTANTE: O AUTISMO ....................................

Indianara Rovêa, Bernardete Pretto

AVIFAUNA NA UNIVERSIDADE DO VALE DO TAQUARI EM

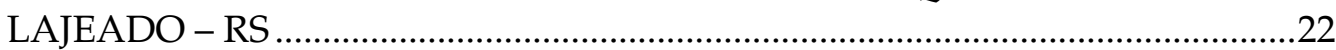

Cleberton Diego Bianchini, Felipe Kuhn, Hamilto Cézar Zanardi Grillo

O AMBIENTE DE CUIDADO EM UTI NEONATAL: A PERCEPÇÃO

DOS PAIS E DA EQUIPE DE SAÚDE .

Paula Michele Lohmann, Eduardo Périco, Luis Felipe Pissaia,

Arlete Eli Kunz da Costa, Claudete Moreschi

A ESPERA NO CENTRO CIRÚRGICO: PERCEPÇÃO DO FAMILIAR 44

Denise Teresinha Arnhold, Paula Michele Lohmann, Luís Felipe Pissaia,

Arlete Eli Kunz da Costa, Claudete Moreschi

COACHING NAS ORGANIZAÇÕES: PESQUISA COM GESTORES E AS APROXIMAÇÕES COM A PSICOLOGIA

Márcia Werner, Claudia Werner Johann

QUALIDADE DE VIDA DE ADOLESCENTES QUE PRATICAM

ATIVIDADE FÍSICA: UMA REVISÃO SISTEMÁTICA .76

Rodrigo Lara Rother, Claudete Rempel

ESQUEMA VACINAL DE HEPATITE B EM PROFISSIONAIS DE

ENFERMAGEM DE UM HOSPITAL DE MÉDIO PORTE

Anelise Carvalho Rauber, Paula Michele Lohmann, Luís Felipe Pissaia,

Arlete Eli Kunz da Costa

ESTÁGIO SUPERVISIONADO: UMA PESQUISA COM ESTUDANTES

DE PSICOLOGIA .96

Cristiane Guaragni, Alice Grasiela Cardoso Rezende Chaves 
O PSICÓLOGO E A UNIDADE DE TERAPIA INTENSIVA (UTI): UM

OLHAR PELA FOTOGRAFIA

Claudimara do Prado, Gisele Dhein

PNEUMONIA LIPOÍDICA E A IMPORTÂNCIA DA HISTÓRIA

CLÍNICA PARA O DIAGNÓSTICO DIFERENCIAL: RELATO DE CASO.....130

Bruna Zagonel, João Henrique Ardenghi Feldens, Dennis Baroni Cruz

PERFIL DA APTIDÃO FÍSICA, DO ESTADO NUTRICIONAL E

DO NÍVEL DE ATIVIDADE FÍSICA DE CRIANÇAS DE ESCOLAS

MUNICIPAIS DA CIDADE DE VENÂNCIO AIRES-RS

Ana Paula Bogorni, Higor Rissini Ferrari, Luiz Gabriel da Silva,

Jéssica Luana Dornelles da Costa, Caroline Pieta Dias, Carlos Leandro Tiggemann

INFLUÊNCIA DO NÍVEL DE ATIVIDADE FÍSICA NA CAPACIDADE

FUNCIONAL DE MULHERES IDOSAS.

Bruno de Maman, Fabian Arruda Barbosa, Augusto Brugnera,

Daniela Beatriz da Silva, Rômulo Augusto Scherer,

Jéssica Luana Dornelles da Costa, Carlos Leandro Tiggemann

A IMPORTÂNCIA DA PESCA COM TIMBÓ PARA O POVO INDÍGENA APYÃWA (TAPIRAPÉ) DE MATO GROSSO.

Xawapa'io Tapirapé, Marcelo Franco Leão

RELATO DE EXPERIÊNCIA: PERCEPÇÃO ACERCA DO SIGNIFICADO

DOS CORTES NO CORPO DO ADOLESCENTE.............................................168

Camila Eleutério Bussmann, Bernardete Pretto

PESQUISA DE COLIFORMES TOTAIS EM ALIMENTOS

COMERCIALIZADOS NO MUNICÍPIO DE JI-PARANÁ, RONDÔNIA ........186

Tiago Barcelos Valiatti, Izabel Bárbara Barcelos,

Estela Nathana Mendonça Sanches, Deise Kelly da Silva,

Gabrielle Melo Calegari, Fernanda Karen Virgolino de Almeida,

Priscila Ferreira Lima dos Prazeres, Wilker Moura Costa Silva,

Natália Faria Romão, Fabiana de Oliveira Solla Sobral

A INFLUÊNCIA DO NÍVEL DE ATIVIDADE FÍSICA NO EQUILÍBRIO EM MULHERES IDOSAS

Fabian Arruda Barbosa, Augusto Brugnera, Bruno De Maman,

Rômulo Augusto Scherer, Daniela Beatriz da Silva,

Jéssica Luana Dornelles da Costa, Caroline Pieta Dias,

Carlos Leandro Tiggemann 
INCIDÊNCIA DE PARASITOS INTESTINAIS EM CRIANÇAS DAS

ESCOLAS MUNICIPAIS DE EDUCAÇÃO INFANTIL E ENSINO

FUNDAMENTAL DE UM MUNICÍPIO DO INTERIOR DO RIO

GRANDE DO SUL

206

Makely Anny Fachini Delazeri, Gabriela kniphoff da Silva Lawisch

O ENSINO DE ARTES MARCIAIS PARA CRIANÇAS: UMA PROPOSTA PEDAGÓGICA

Marcelo Rossetto, Derli Juliano Neuenfeldt

O CUIDAR EM ONCOLOGIA PEDIÁTRICA: UM ESTUDO BASEADO

NO PROCESSO DE ENFERMAGEM

Mary Elizabeth de Santana, Elanny Glicia Oliveira da Costa,

Anderson Roberto de Sales Corrêa, Wagner Luiz Oliveira Ximenes

RESPOSTAS DA FREQUÊNCIA CARDÍACA DE JOVENS ATLETAS DE MODALIDADES COLETIVAS APÓS TESTES DE DESEMPENHO

Algicelia Sousa Ponte Guimarães, Vitória Andrade Araújo,

Thainá Costa Barreto, Lucas Guilherme Torres Ferreira,

Ricardo Alexandre Rodrigues Santa Cruz

AVALIAÇÃO DA ATIVIDADE LEISHMANICIDA IN VITRO DE ESPÉCIES DA FAMÍLIA MYRTACEAE, NATIVAS DO SUL DO BRASIL....246

Carla Kauffmann, Leandra Andressa Pacheco, Bárbara Buhl, Talita Scheibel, Elisete Maria de Freitas, Lucélia Hoehne, Gérzia Maria de Carvalho Machado, Marilene Marcuzzo do Canto Cavalheiro, Simone Cristina Baggio Gnoatto, Eduardo Miranda Ethur

O FARMACÊUTICO EM SERVIÇO DE ATENÇÃO SECUNDÁRIA À SAÚDE: ATUAÇÃO EM EQUIPE MULTIPROFISSIONAL PARA PROMOÇÃO DO USO RACIONAL DE MEDICAMENTOS

Alana Lansing, Juliana de Souza, Luciana Carvalho Fernandes,

Luís César de Castro, Carla Kauffmann

PRINCIPAIS CARACTERÍSTICAS DAS GESTANTES DE ALTO RISCO DA REGIÃO CENTRAL DO RIO GRANDE DO SUL

Paula Fernanda Jantsch, Ioná Carreno, Adriane Pozzobon, Fernanda Scherer Adami, Carolina de Souza Leal, Ticiane Codevila da Silva Mathias, Amanda Savaris Ludwig, Pedro Henrique Fernandes Bergo

VARIAÇÕES NO MICROCLIMA E CARACTERÍSTICAS DO SOLO EM PAISAGENS COM DIFERENTES COBERTURAS VEGETAIS: AÇÃO DE CAMPO JUNTO AO MORRO DA HARMONIA - TEUTÔNIA/RS

Melissa Heberle, Berenice Maria Dalla Costa da Silva, Charlyan de Souza Lima,

Renato Santiago Quintal, Claudete Rempel, Marina Schmidt Dalzochio 